\title{
LImits to ion energy control in high density glow discharges: Measurement of absolute metastable ion concentrations
}

\author{
Konstantinos P. Giapis, Nader Sadeghi, ${ }^{\text {a) }}$ Joëlle Margot, ${ }^{\text {b) }}$ Richard A. Gottscho, \\ and T. C. John Lee \\ AT\&T Bell Laboratories, Murray Hill, New Jersey 07974
}

(Received 8 September 1992, accepted for publication 2 February 1993)

\begin{abstract}
Unprecedented demands for uniformity, throughput, anisotropy, and damage control in submicron pattern transfer are spurring development of new, low pressure, high charge density plasma reactors. Wafer biasing, independent of plasma production in these new systems is intended to provide improved ion flux and energy control so that selectivity can be optimized and damage can be minimized. However, as we show here, an inherent property of such discharges is the generation of significant densities of excited, metastable ionic states that can bombard workpiece surfaces with higher translational and internal energy. Absolute metastable ion densities are measured using the technique of self-absorption, while the corresponding velocity distributions and density scaling with pressure and electron density are measured using laser-induced fluorescence. For a low pressure, helicon-wave excited plasma, the metastable ion flux is at least $24 \%$ of the total ion flux to device surfaces. Because the metastable ion density scales roughly as the reciprocal of the pressure and as the square of the electron density, the metastable flux is largest in low pressure, high charge density plasmas. This metastable ion energy flux effectively limits ion energy and flux control in these plasma reactors, but the consequences for etching and deposition of thin films depend on the material system and remain an open question.
\end{abstract}

\section{INTRODUCTION}

Because of shrinking dimensions in the manufacture of integrated circuits, unprecedented demands have been placed on plasma etching processes that are used to transfer elements of the circuit from a lithographically dcfined pattern to underlying materials such as $\mathrm{Si}$, poly-Si, $\mathrm{Al}$, and $\mathrm{SiO}_{2}{ }^{1,2}$ For example, a tolerance of $10 \%$ in gate linewidth for a $0.25 \mu \mathrm{m}$ design rule means that the plasma etching process must be controlled to within $25 \mathrm{~nm}$. Similarly, as gate oxide thicknesses shrink below $10 \mathrm{~nm}$ for ultralarge scale integrated (ULSI) circuits, Si-gate etch processes that terminate on such oxides must be extraordinarily selective since overetches of $100 \%$ are common practice. Since larger wafers are now being processed one at a time uniformity must be improved to minimize overetch time and etching rates must be increased to maintain high throughput.

For these reasons, new plasma sources and reactors are being developed. ${ }^{3-8}$ Such sources must operate at lower pressure to maintain critical dimension control but with higher charge density to maintain high throughput: thus, the degree of ionization must be increased beyond that used in conventional rf diode reactors. To optimize selectivity and minimize ion-induced damage, the average ion energy must be tuned to just above threshold for reactive ion etching while remaining below threshold for producing damage. ${ }^{9}$ Of course, average ion energy is not the only

\footnotetext{
a) Permanent address: Laboratoirc de Spectrometrie Physique, University Joseph Fourier and CNRS, Grenoble, France.

b) Permanent address: Department of Physics, University of Montreal, Montreal, Canada.
}

pertinent parameter; the ion energy-both translational and internal-must be narrowly distributed about this average value. Traditional radio frequency diode systems do not provide sufficient control to meet the above criteria so attention has focused on a class of wave-excited plasma reactors: for example, electron cyclotron resonance (ECR), helicon mode, and helical resonator. In each of thcse, the workpiece is placed on an electrode that is independently biased with respect to the power source used to generate the plasma; the intention is to achieve superior control of ion flux and energy.

If one examines the above criteria more closely, however, inherent limitations and tradeoffs become apparent. For example, generating high plasma density at low pressure necessarily results in larger ultraviolet (and in some cases $\mathrm{x}$-ray) emission that can affect device yield and reliability. ${ }^{10-13}$ Similarly, efficient plasma generation at low pressure must produce a distribution of excited ionic states and doubly ionized atoms both of which can effectively broaden the ion energy distributions, both internally and translationally, and again device yield and reliability may be affected. The creation and destruction of metastable ionic states and measurement of their absolute density is the subject of this work.

Previously, ${ }^{14-16}$ we sampled metastable ion velocity distribution functions in $\mathrm{Ar}$ and $\mathrm{Cl}_{2}$ plasmas using the laser-induced fluorescence (LIF) technique. Similar measurements have been made on ground state $\mathrm{N}_{2}^{+}$formed in an ECR plasma at the University of Wisconsin. ${ }^{17}$ Several conclusions were drawn from these measurements:

(i) Commensurate with the specifications for linewidth control, the inherent, thermal broadening of both ground 
state and metastable state ion velocity distribution functions (ivdfs) is relatively small.

(ii) Plasma expansion, e.g., in a downstream configuration, can produce radial acceleration of both ground state and metastable state ions and compromise both linewidth control and etching uniformity. The nonuniform ion flux may also aggravate charge-induced damage to thin gate oxides. ${ }^{18-20}$

(iii) As a result of reduced charge exchange collisionality, metastable state ions typically have larger averagc $(\sim 10 \mathrm{eV})$ ion energy and broader ion energy distributions than their ground state counterparts. Although this higher translational energy is relatively small compared to the energy gained by acceleration in the sheath field, it may be significant if operation near threshold is desired.

In addition to excess translational energy, metastable ions in $\mathrm{Ar}$ and $\mathrm{Cl}_{2}$ plasmas carry more than 16 and $13 \mathrm{eV}$, respectively, of internal, electronic energy. Even with this excess energy--translational and electronic-the metastables may play little role in surface modification if their flux to the surface is small compared to the ground state ion flux. The key question is: What is the flux of metastable ions relative to ground state ions and how does this flux scale with plasma parameters? To answer these questions, we measure absolute densities of 5 metastable ionic states of Ar in a helicon-wave excited plasma using the technique of self-absorption. To place an upper bound on the ground state ion density $n_{i}$, and determine the fraction of ions in metastable states, we use microwave interferometry to measure the electron density, $n_{e}$. For the $\mathrm{Ar}^{+}\left(3 p^{4} 3 d^{2} G_{9 / 2}\right)$ level, we use LIF to determine how the metastable density, $n_{m}$, scales with $n_{e}$ and gas pressure. We concentrate our efforts on the Ar plasma for two reasons: (1) the data base is better developed for Ar than it is for processing gases such as $\mathrm{Cl}_{2}$ and this should facilitate quantitative comparisons between simulation and experiment and (2) our previous measurements on $\mathrm{Cl}_{2}$ plasmas indicate that the ion dynamics are similar to those found in the corresponding Ar plasmas. ${ }^{14-16}$

\section{EXPERIMENTAL DETAILS}

\section{A. Helicon reactor}

The principles underlying helicon wave excitation of plasmas can be found in the literature. ${ }^{21-27}$ The plasma reactor and experimental configuration used here are similar to those used previously in diagnosing the source and reactor regions of ECR and helicon-wave driven plasmas. ${ }^{14-16}$ The magnetic field configuration employed in the source is identical to that reported previously ${ }^{14}$ except that the magnitude is reduced by 2 times. Flow rates are regulated using mass flow controllers but the pressure is not actively controlled.

Radio frequency power at $13.56 \mathrm{MHz}$ from a Plasma Therm HFS 2500D generator is applied to an antenna through an impedance matching network as shown in Fig. 1. If power is measured using the built-in power meter of the generator and it should be noted that some power is

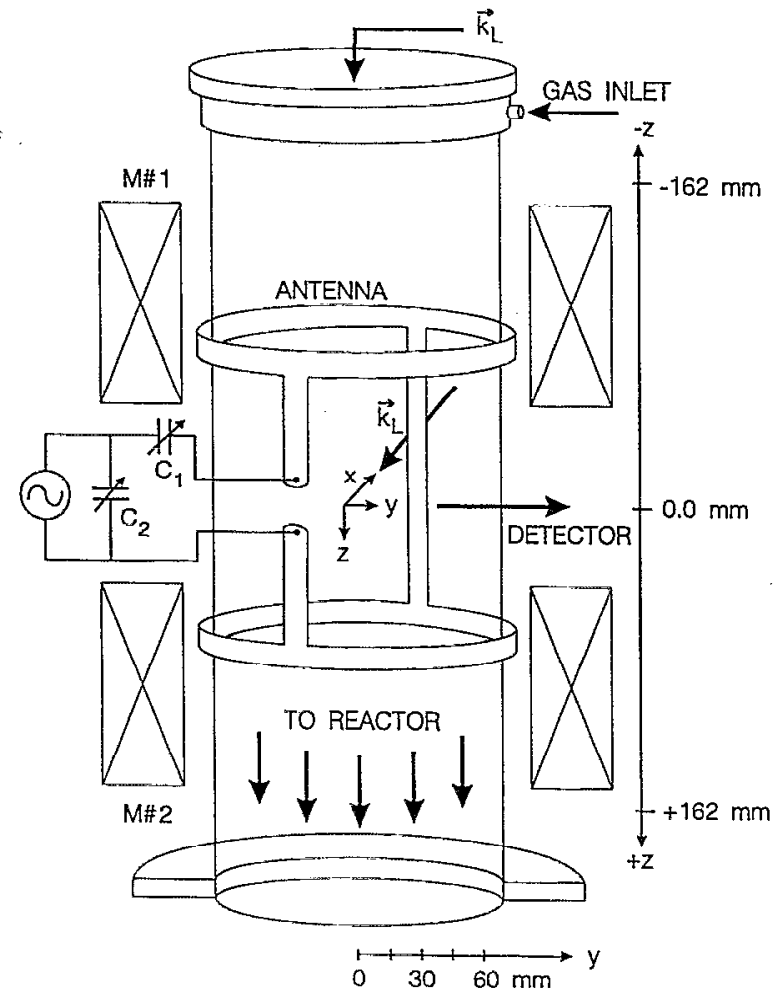

FIG. 1. Schematic illustration of helicon reactor with antenna supplied by Lucas Labs (aftcr modification) and corresponding power supply and impedance matching network. The diagnostic geometry and axes definitions are shown for the laser-induced fluorescence experiments.

dissipated in the matching network, necessitating aircooling.

\section{B. Laser-induced fluorescence measurements}

We use a $1 \mathrm{MHz}$ linewidth, continuous wave, $\mathrm{Ar}^{+}$. pumped, single mode dye laser (Coherent 699-29) to excite the $4 p^{\prime 2} F_{7 / 2}^{0} \leftarrow 3 p^{4} 3 d^{2} G_{9 / 2}$ transition in $\mathrm{Ar}^{+}$at 611.49 $\mathrm{nm}$. The laser system includes an integral wave meter, calibrated against the molecular iodine atlas, ${ }^{28}$ with which we measure wavelength shifts to $\pm 10^{-4} \mathrm{~nm}$. After passing through $130 \mathrm{~m}$ of AT\&T $62.5 \mu \mathrm{m}$ core diameter multimode fiber to bring the beam to the helicon system in a nearby laboratory, the unpolarized laser beam is chopped. The $4 p^{\prime 2} F_{7 / 2}^{0} \rightarrow 4 s^{\prime 2} D_{5 / 2}$ transition is detected in fluorescence at $460.96 \mathrm{~nm}$.

Using a $75 \mathrm{~mm}$ focal length, 50-mm-diam lens, fluorescence is collected at right angles to the laser propagation direction and focused onto a slit-shaped fiber-optic bundle (General Fiber Optics). The other end of the bundle is also slit shaped and imaged onto the entrance slit of a $1.25 \mathrm{~m}$ scanning monochromator (SPEX 1269) equipped with a Hamamatsu R376 photomultiplier tube, whose output is fed into a phase-locked amplifier. Spatial resolution is dictated by bundle dimensions. lens focal length, and lens placement: $\Delta z \approx 1 \mathrm{~mm}$ and $\Delta x \approx 5 \mathrm{~mm}$. Because of the finite laser beam diameter, the resolution along the detector direction is $\Delta y \approx 2 \mathrm{~mm}$. 


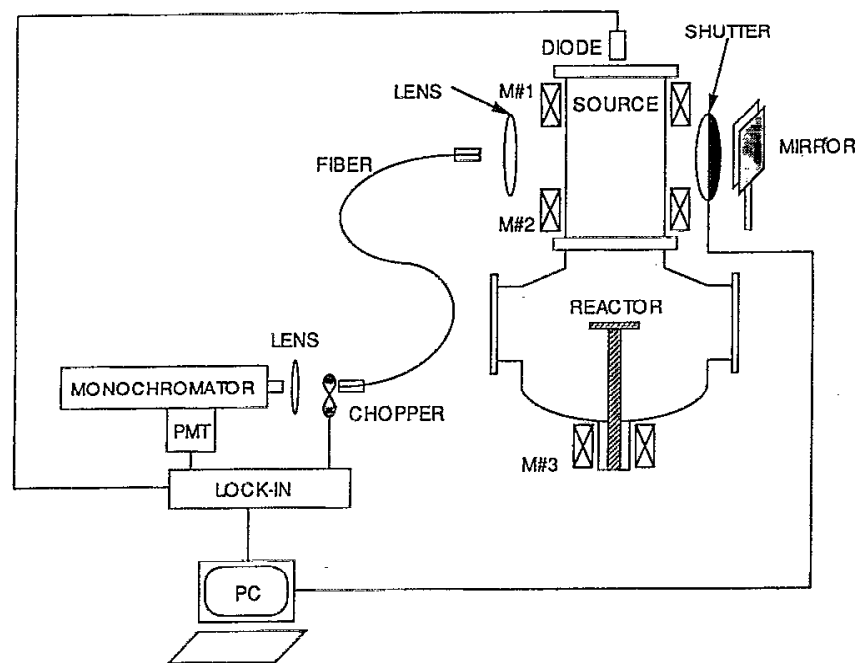

FIG. 2. Schematic illustration of helicon-wave excited plasma reactor showing self-absorption setup and data collection system.

\section{Self-absorption measurements}

Optical absorption is a relatively simple method for making absolute density measurements. ${ }^{29,30}$ Light sources used for these measurements include a continuum emitting lamp, ${ }^{31,32}$ for example a high pressure xenon discharge or a tungsten filament, a resonance line lamp containing the same atoms as in the absorbing medium, ${ }^{29,30}$ and a laser tuned to the absorption line. ${ }^{33,34}$ Advantages of using the resonance absorption technique rather than the two others are: (1) linewidths for absorption and emission are generally well matched thus making the technique more sensitive and (2) the entire velocity distribution in the Doppler profile of the absorbing atoms is simultaneously accessible making the method simpler to implement than those based on tuning a narrow bandwidth laser. Resonance probe lamps have been widely used for monitoring the density of the atoms in their ground and metastable states. ${ }^{29,30,35}$ However, in highly ionized plasmas such as ECR and helicon sources, the emission intensities of ionic lines are quite strong while in the usual resonance lamps the electronic temperature is much lower, making the ionic line intensities too weak for sensitive absorption measurements. Therefore, we use the helicon plasma itself as the probing light source for absorption. Excited state emission from the plasma is directed back into the reactor and detected after being absorbed (Fig. 2).

We collect light along a column through the plasma by positioning the slit-shaped fiber-optic bundle in the focal plane of a $100 \mathrm{~mm}$ focal length, 50-mm-diam lens. Using a shutter to block light from the back mirror, we detect a single pass intensity $I_{1}$; with retroreflection, we detect a total intensity

$$
I_{T}=I_{1}+I_{2}=I_{1}[1+R(1-A)],
$$

where $A$ is the fractional absorption of the radiation after crossing the plasma and $R$ is the effective reflectivity of the mirror, which can include scattering and absorption losses
TABLE I. Ar ion metastable densities in the $3 p^{4} 3 d$ manifold $^{\mathrm{n}}$

\begin{tabular}{lccccl}
\hline $\begin{array}{c}\text { State } \\
(\mathrm{eV})\end{array}$ & $\begin{array}{c}\text { Energy } \\
(\mathrm{nm})\end{array}$ & $\begin{array}{c}\text { Transition } \\
\text { strength }\end{array}$ & $\begin{array}{c}\text { Oscillator } \\
(\%)^{\mathrm{b}}\end{array}$ & $\begin{array}{c}\text { Absorbance } \\
\left(\mathrm{cm}^{-3}\right)\end{array}$ \\
\hline${ }^{4} D_{7 / 2}$ & 16.4 & 440.10 & 0.066 & 9.1 & $>1.4 \times 10^{10}$ \\
${ }^{4} D_{3 / 2}$ & 16.4 & 440.01 & 0.046 & 5.2 & $>1.2$ \\
${ }^{4} D_{1 / 2}$ & 16.5 & 435.22 & 0.060 & 0.5 & $>0.08$ \\
${ }^{4} F_{9 / 2}$ & 17.6 & 664.37 & 0.078 & 14.2 & $>1.2$ \\
${ }^{2} G_{9 / 2}$ & 19.1 & 611.49 & 0.090 & 12.3 & $>1.0$ \\
\hline \hline
\end{tabular}

${ }^{a}$ Reference line used for all measurements was $475.29 \mathrm{~nm} ; R=1.97 \pm 0.01$. All measurements were performed on $0.067 \mathrm{~Pa}(0.5 \mathrm{mTorr})$ and $1 \mathrm{~kW}$ nominal if power. Oscillator strengths calculated from data in Ref. 16; $T_{A}=2500 \mathrm{~K}, T_{E}=0 \mathrm{~K}$, and $L=15 \mathrm{~cm}$ assumed.

${ }^{b}$ Absorbance values are precise to $\pm 1 \%$.

on the back window and mirror, anisotropy in the spatial dependence of the plásma-induced emission, as well as the true mirror reflectivity. Light emanating from the other end of the fiber-optic bundle is first chopped and then imaged onto the entrance slit of the spectrometer. A phase sensitive amplifier is used to detect the signal from the photomultiplier tube. To reduce sensitivity to low frequency fluctuations of the emission intensity from the plasma, the signal is normalized to that obtained from a silicon photodetector that collects spectrally unresolved plasma emission. A computer is used to collect data alternately with the shutter open and then closed. To determine the value of $R$ in Eq. (1), we measure $I_{1}$ and $I_{T}$ for a nonabsorbing line $(A=0)$ or two absorbing lines that terminate in the same lower level and for which the oscillator strengths are both known. Using a nonabsorbing reference line is generally preferred since the propagation of errors is minimized.

Given absorption for a particular line, we need to deduce the absolute lower state density. To do so, we first assume that both the emission and absorption profiles of the line are Gaussian (Doppler broadened) and that the absorption is weak (i.e., $A \lesssim 0.15$ ) in which case, for $\mathrm{Ar}$ atoms or ions ${ }^{29,30}$

$$
N=1.36 \times 10^{12} A \frac{\sqrt{T_{E}+T_{A}}}{L f \lambda},
$$

where $N$ is the line-averaged lower level density in $\mathrm{cm}^{-3}, A$ is the absorbance deduced from Eq. (1), $T_{E}$ and $T_{A}$ are the temperatures in $\mathrm{K}$ corresponding to the emitting and absorbing ions in the upper and lower levels of the transition, respectively, $L$ is the path length in $\mathrm{cm}, f$ is the oscillator strength ${ }^{36}$ and $\lambda$ is the central wavelength of the transition in $\mathrm{nm}$. The weak absorption assumption means that we can ignore line-of-sight variation in the Doppler profile of the back reflected optical signal, as well as the spatial inhomogeneities in the absorption profile. For the strongest absorbing lines where $A \sim 0.14$, the weak absorption assumption introduces an error of $\sim 2 \%$, which is comparable to our experimental precision (Table I). Assuming a flat metastable $\mathrm{Ar}^{+}$profile (Fig. 3), we take $L=15 \mathrm{~cm}$.

Consistent with the assumptions used in deriving Eq. (2), we know from the LIF measurements that the absorbing line profile for the $\operatorname{Ar}^{+}\left(3 p^{4} 3 d^{2} G_{9 / 2}\right)$ transition is 


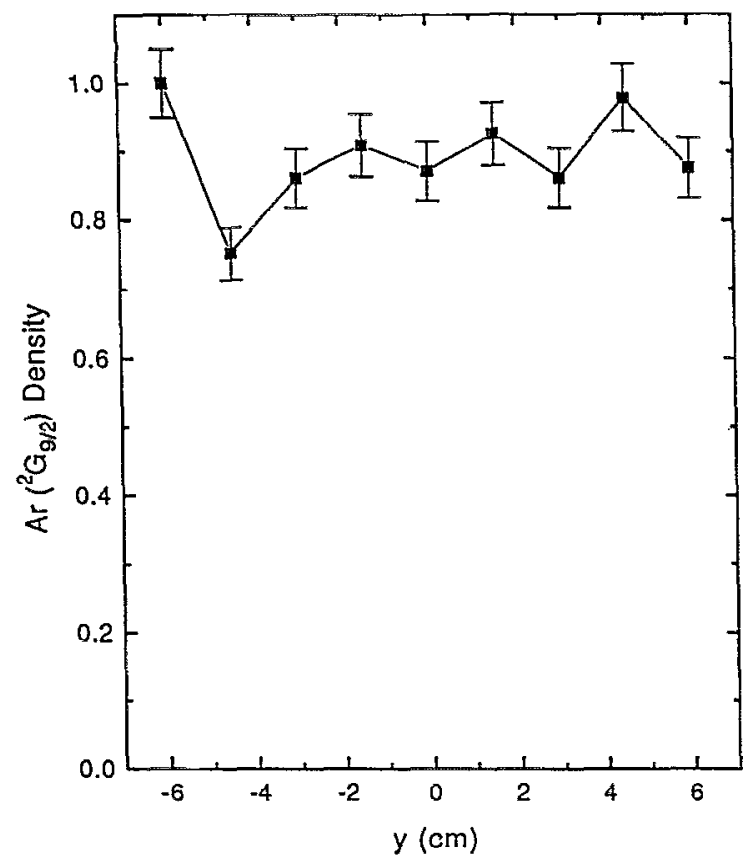

FIG. 3. Spatial profile of $\operatorname{Ar}^{+}\left(3 p^{4} 3 d^{2} G_{9 / 2}\right)$ density at $0.13 \mathrm{~Pa}$ and $1 \mathrm{~kW}$, $x=z=0$, corresponding to line-of-sight self-absorption measurements. The $f\left(v_{z}\right)$ distribution is measured and then integrated at various $y$ positions to generate the plot shown.

nearly Gaussian over most of the radius, although the lines broaden and shift slightly near the walls of the reactor (Fig. 4). If we assume the emitting state shifts in an identical fashion we generate a lower bound to $N$; any other assumption only increases our estimation of $N$. For example, if the emitting state does not shift at all, the overlap between the line profiles for absorbing state and emitting state will be smaller; and, for the same absorption, a larger metastable density would be deduced. Because the absolute density deduced for a measured absorption depends only on the square root of the absorbing level's temperature [Eq. (2)], we ignore the broadening that occurs toward the walls and generate a lower bound estimate to $N$. Similarly, we make the conservative assumption that $T_{E}<T_{A}$ and again place a lower bound on the determination of $N$.

\section{Electron density measurements}

To compare metastable ion densities to the total charge density, we estimate line-integrated electron densities from the phase shift of a $35 \mathrm{GHz}$ microwave interferometer (PMT DDM-01-B). To obtain an estimate of $n_{e}$ we assume a flat profile, which is consistent with the metastable ion profile shown in Fig. 3, and simply divide the lineintegrated values by the effective path length of $15 \mathrm{~cm}$. All measurements are made through ports in the center $(z=0)$ of the ground shield around the rf antenna along the $y$ axis (Fig. 1). The influence of magnetic fields on the phase shift can be ignored since the electron cyclotron frequency is much lower than the interferometer wave frequency.

We note that the phase shifts measured vary by as much as a factor of 2 , as the distance between the receiving

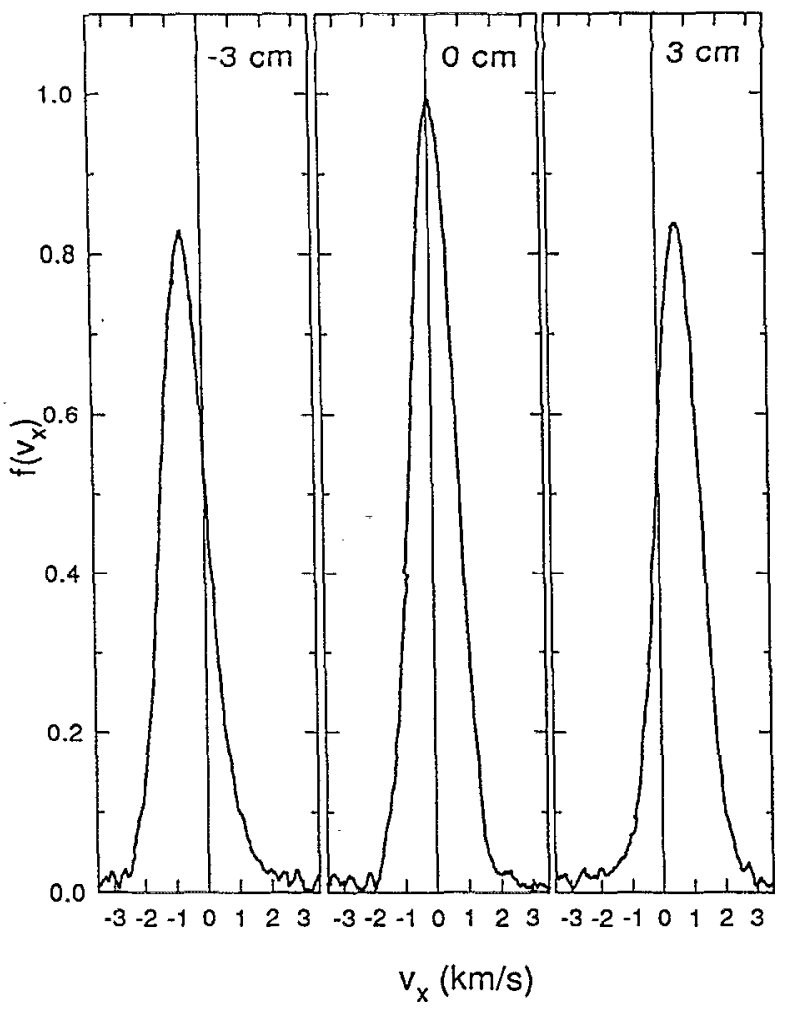

FIG. 4. Ion velocity distribution functions, $f\left(v_{x}\right)$, at $z=y=0$ and 3 different values of $x$ as shown. The Ar pressure is $0.13 \mathrm{~Pa}$ and the rf power is $1 \mathrm{~kW}$.

horn and the reactor wall are varied. We attribute this variation to reflections from the metal shield surrounding the quartz reactor tube and take the largest value measured to continue placing a lower bound on $n_{m}$ relative to $n_{i}$.

\section{METASTABLE DENSITIES}

Line-averaged metastable densities for five levels are given in Table $\mathrm{I}$ for a pressure of $0.067 \mathrm{~Pa}(0.5 \mathrm{mTorr})$ and $1.0 \mathrm{~kW}$ nominal power. These estimates are lower bounds for the reasons enumerated above. Moreover, their sum is a lower bound estimate to the total metastable and nonmetastable excited ionic state density since other metastable levels with $3 p^{4} 3 d$ valence configuration $\left({ }^{4} D_{5 / 2}\right.$, ${ }^{4} F_{7 / 2,5 / 2,3 / 2},{ }^{4} G_{11 / 2,9 / 2,7 / 2,5 / 2}$, and $\left.{ }^{2} G_{7 / 2}\right)^{37}$ are surely populated although their densities have not been measured because of interference with strong transitions of the neutral Ar atom.

To compare $n_{m}$ shown in Table I to the total charge density, we use the microwave interferometer data of Fig. 5. For the specific conditions given in Table I, the overall metastable ion density of the five levels monitored is larger than $4.8 \times 10^{10} \mathrm{~cm}^{-3}$ and the total ion density (approximately equal to $n_{e}$ ) given in Fig. 5 is at most $2 \times 10^{11} \mathrm{~cm}^{-3}$. Therefore, we find at least $24 \%$ of all ions reside in excited, metastable states with more than $16 \mathrm{eV}$ of internal energy. Since these ions have higher velocity normal to the surface, the metastable ion flux will be more than $24 \%$ of the total 


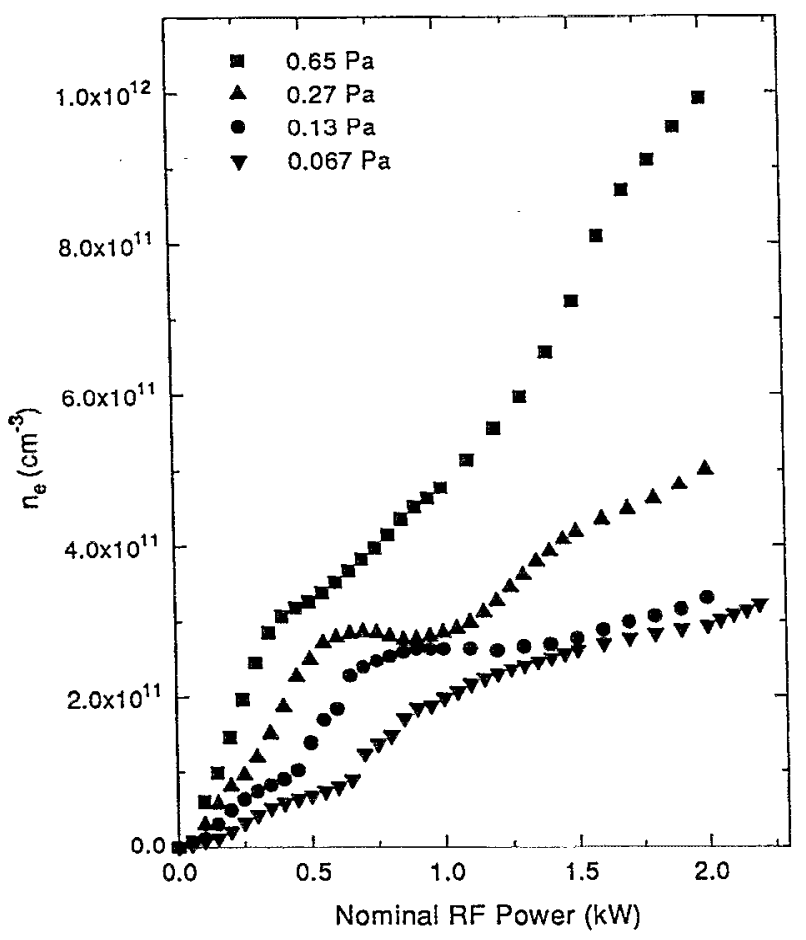

FIG. 5. Electron density as a function of if power for several Ar pressures deduced from microwave interferometer phase shifts and the assumption that the spatial profile is uniform.

ion flux when surfaces are placed in the source region of the reactor, which is now common practice for ECR systems. ${ }^{38,39}$

It is important to know how the metastable density and flux vary with plasma parameters. This knowledge provides insight into the formation and loss mechanisms and helps to explain why metastables are so abundant. More importantly, such scaling relationships enable prediction and control of metastable ion energy flux for materials processing applications. Unfortunately, the signal to noise ratio in the absorption experiments precludes making such determinations. At higher pressures the metastable density decreases (Fig. 6) while at higher powers, fluctuations in the plasma emission intensity limit accurate selfabsorption measurements. So, we turn to LIF measurements for accurate scaling of the $\operatorname{Ar}^{+}\left(3 p^{4} 3 d^{2} G_{9 / 2}\right)$ level. Whether or not all metastable levels scale in the same way remains an open question.

Figure 6 shows that the $\operatorname{Ar}^{+}\left(3 p^{4} 3 d^{2} G_{9 / 2}\right)$ level density decreases dramatically as the gas pressure is increased. These measurements are made with the laser propagating along $\hat{x}$ and the detection optics centered at either $z=0$ or $-16.5 \mathrm{~cm}$. The measured $f\left(v_{x}\right)$ distributions are integrated to obtain relative density estimates, whose scaling with pressure is approximately independent of both position and microwave power. If the metastable ion formation rate were independent of pressure and the dominant loss mechanism were collisional quenching of the metastable state by collisions with the neutral atoms, a $1 / P$ scaling of

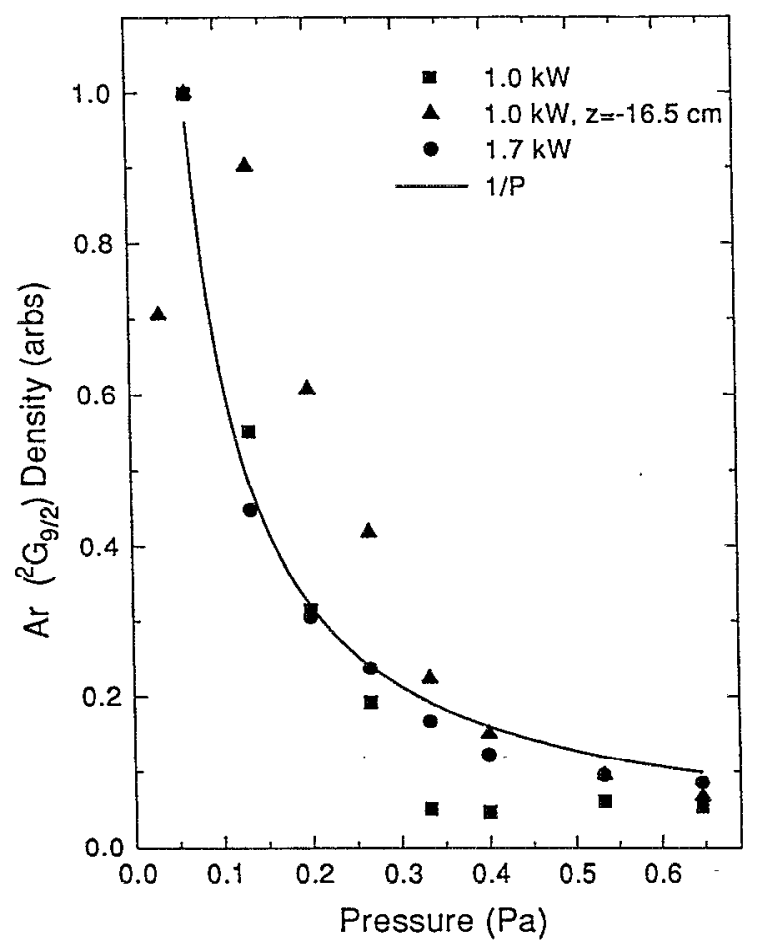

FIG. 6. $\mathrm{Ar}^{+}\left(3 p^{4} 3 d^{2} G_{9 / 2}\right)$ density as a function of pressure and $\mathrm{rf}$ power at $x=y=z=0$. The $f\left(v_{x}\right)$ ivdf is measured and integrated for each power and pressure.

the density (Fig. 6) would be expected. Penning ionization,

$$
\mathrm{Ar}_{m}^{+}+\mathrm{Ar} \rightarrow \mathrm{Ar}^{+}+\mathrm{Ar}^{+}+e^{-}
$$

is a likely quenching mechanism since it is nearly energy resonant and the apparent cross section is large; ${ }^{15}$ thus, the mean free path at the highest pressures is much less than the reactor tube radius. This mechanism would also contribute to the observed increase in $n_{e}$ as the pressure is increased from 0.067 to $0.67 \mathrm{~Pa}$ (Fig. 5). Clearly, the density dependence on pressure is not so simple that only quenching can be considered: the errors associated with each of the points in Fig. 6 are much less than the deviations from the $1 / P$ curve. These deviations signal a change, generally a decrease, in the production rate of the metastables as the pressure is increased. Nonetheless, the decrease in $n_{m}$ with increasing pressure is consistent with quenching being an important loss mechanism. While wall loss is certainly important at the lowest pressures, this mechanism should only be weakly pressure dependent and become relatively unimportant as the pressure is increased. The increase in $n_{e}$ as pressure is increased (Fig. 5) will tend to compensate for the expected decrease in electron temperature, $T_{e}{ }^{40}$ so that the metastable ion formation rate will vary only weakly with pressure and the approximate $1 / P$ scaling (which results from loss by quenching) becomes apparent. In any case, operation at higher pressures, all else being equal, will tend to reduce the metastable to ground state ion flux ratio. 


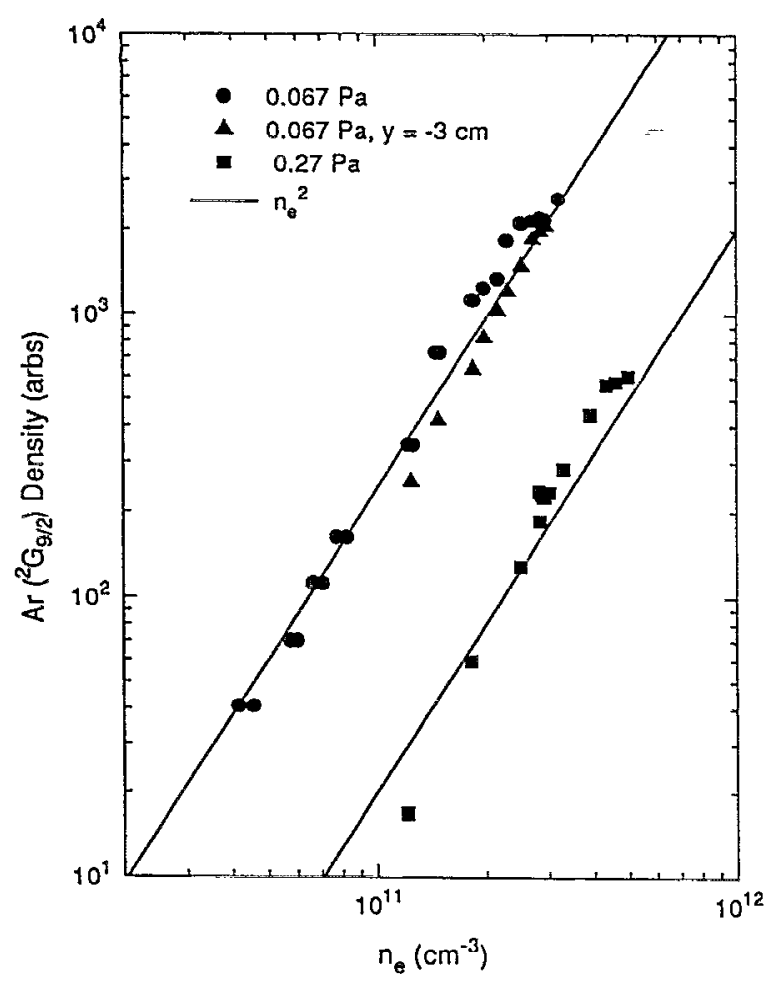

FIG. 7. $\mathrm{Ar}^{+}\left(3 p^{4} 3 d^{2} G_{9 / 2}\right)$ density as a function of electron density for two pressures and positions. The electron densities are measured interferometrically (Fig. 5) and varied by changing if power only. The data are obtained at $x=y=z=0$ except for the triangles where $y=-3 \mathrm{~cm}$.

When rf power is increased at constant pressure, the electron density also increases (Fig. 5). The corresponding change in the $\mathrm{Ar}^{+}\left(3 p^{4} 3 d^{2} G_{9 / 2}\right)$ level density as determined by LIF is shown in Fig. 7. For comparison, a $n_{e}^{2}$ dependence is also shown illustrating the close correspondence, particularly at the lower pressure; this scaling relationship is approximately independent of position in the plasma and gas pressure. ${ }^{41}$ Thus, we conclude that metastable ions are formed predominantly by a 2 -electron process, for example ionization of metastable neutrals ${ }^{42}$ and/or excitation from ground state ions. All else being equal, the metastable ion to ground state ion flux ratio will increase dramatically as the charge density is increased. Calibrating our previous measurements of ion metastables in a higher density ECR plasma, we find good agreement with the data in Figs. 6 and 7, suggesting that the scaling may be independent of plasma excitation mode.

At first, it may seem odd that $n_{m}$ is such a large fraction of $n_{i}$. However, consideration of the phase space occupied by these different states is illuminating. The two spin components of $\mathrm{Ar}^{+}$ground state have ${ }^{2} P_{3 / 2}$ and ${ }^{2} P_{1 / 2}$ symmetry corresponding to a total degeneracy $g=6$. By contrast, the doublet and quartet metastable levels listed in Table I have a combined degeneracy of $g=34$ out of a total degeneracy of $g=102$ for the metastable ion manifold $\left(3 p^{4} 3 d^{4} D,{ }^{4} F,{ }^{4} G,{ }^{2} G\right) \cdot{ }^{37}$ This gives a level degeneracy ratio of $17: 1$ for the metastable ions relative to the ground state ions. In other words, once the ground state ion is formed using a minimum of $15.8 \mathrm{eV}$, electrons with com- parable energy have a statistically excellent opportunity to form an energy reservoir in the metastable ion state manifold.

If the electronic states of the ion and neutral argon were in equilibrium with the free electrons in the plasma and if we consider that the observed metastable ion states $(g=34)$ contain $1 / 3$ of the total metastable ion population $(g=102)$; then the ratio of the total metastable ions to the ground state ions is

$$
0.6 / 0.4=(102 / 6) \exp \left(-18 / T_{e}\right),
$$

or $T_{e} \approx 7.5 \mathrm{eV}$. While this value for $T_{e}$ seems reasonable, we caution that the data in Table I clearly show that the metastable ions are not in equilibrium with the free electrons in the plasma-note the anomalously small population in the ${ }^{4} D_{1 / 2}$ level - and the assumption that we observe only $1 / 3$ of all the metastable ions cannot be justified.

\section{CONCLUSIONS}

We have determined a lower bound on the absolute metastable Ar ion densities in a helicon-wave excited plasma using the technique of self-absorption spectroscopy. Combined with microwave interferometric measurements of electron density, we find that at least $24 \%$ of the Ar ions are in metastable states at $0.067 \mathrm{~Pa}$ and $1 \mathrm{~kW}$ nominal power. Using laser-induced fluorescence spectroscopy on one of the metastable levels, we find that metastable density scales roughly as $n_{e}^{2}$ and $P^{-1}$, where $n_{c}$ is the electron density and $P$ is the total gas pressure. The scaling with electron density is consistent with 2-step formation, for example by ionization and then excitation, while the pressure scaling is consistent with loss by collisional quenching at higher pressures. Relative metastable density measurements made in an ECR reactor are consistent with these observations suggesting that the scaling may be independent of plasma excitation mode.

The large density of metastable ionic states may play an important role in materials processing. At least these states will limit the degree to which the ion energy flux to device wafers can be controlled. Metastable ions tend to have larger average translational energy and broader energy distributions than their ground state ion counterparts as a result of lower charge exchange collisionality. In addition, they have substantial internal electronic energy, $>16 \mathrm{eV}$ in the case of Ar. Operating at lower pressure and higher charge density only increases the relative proportion of metastable states and may further compromise selectivity and yield. The precise magnitude of the effects, which metastable ion bombardment may have, depends on the specific process and in particular how the process scales with ion energy and flux. If the dependence is linear, then these effects may be relatively minor; but if the process is (exponentially) activated, the effects may be dramatic. This dependence remains an open question.

Although the measurements reported here are for Ar gas in a helicon-wave excited plasma, the results should be widely applicable to different gases, excitation modes, and reactor configurations. Our previous work ${ }^{9,11}$. has shown, 
for example, that the metastable ion transport in $\mathrm{Cl}_{2}$ is similar to that in $\mathrm{Ar}$ and we find here that the results scale from our helicon system to our ECR system. However, for $\mathrm{Cl}^{+}$ions the total degeneracy of the metastable states is only 36 compared to a ground state ion degeneracy of 9 , so the ratio is only $4: 1$ compared to $17: 1$ for argon ions. Therefore compared to Ar plasmas, we expect the metastable ion density in $\mathrm{Cl}_{2}$ plasmas to be a smaller fraction of the total ion density.

\section{ACKNOWLEDGMENTS}

Travel and living support for J. Margot was kindly provided through a grant from the Ministère des Affaires Internationales de Québec. Discussions with J. Lawler and C. Woods from the University of Wisconsin and M. Lieberman from U. C. Berkeley are gratefully acknowledged. We are also grateful to Lucas Labs for providing a helicon antenna which we modified slightly and to F. Skiff of the University of Maryland for bringing Ref. 36 to our attention and for many stimulating conversations.

${ }^{1}$ R. B. Fair, Proc. IEEE 78, 1687 (1990).

${ }^{2}$ J. M. Cook and K. G. Donohoe, Solid State Technol. 34, 119 (1991).

${ }^{3}$ K. Suzuki, S. Okudaira, and I. Kanomata, J. Electrochem. Soc. 126,

1024 (1979); K. Suzuki, K. Ninomiya, S. Nishimatsu, and S. Okudaira,

J. Vac. Sci. Technol. B 3, 1025 (1985).

${ }^{4}$ J. Asmussen, J. Vac. Sci. Technol. A 7, 883 (1989) and references therein.

${ }^{5}$ R. W. Boswell, A. J. Perry, and M. Emami, J. Vac. Sci. Technol. A 7, 3345 (1989).

${ }^{6}$ J. M. Cook, D. E. Ibbotson, P. D. Foo, and D. L. Flamm, J. Vac. Sci. Technol. A 8, 1820 (1990).

${ }^{7}$ R. R. Burke and C. Pomot, Solid State Technol. 31 (Feb.), 67 (1988).

${ }^{8}$ K. . . H. Kretschmer, K. Matl, G. Lorenz, I. Kessler, and B. Dumbacher, Solid State Technol. 33 (Feb.), 53 (1990).

${ }^{9}$ W. M. Holber and J. Forster, J. Vac. Sci. Technol. A 8, 3720 (1990).

${ }^{10}$ J. B. O. Caughman II and W. M. Holber, J. Vac. Sci. Technol. A 9, 3113 (1991).

${ }^{11}$ T. Yunogami, T. Mizutani, K. Suzuki, and S. Nishimatsu, Jpn. J. Appl. Phys. 28, 2172 (1989).

${ }^{12}$ T. J. Castagna, J. L. Shohet, K. A. Ashtiani, and N. Hershkowitz, Appl. Phys. Lett. 23, 2856 (1992).

${ }^{13}$ D. A. Buchanan and G. Fortuno-Wiltshire, J. Vac. Sci. Technol. A 9 , 804 (1991).

${ }^{14}$ T. Nakano, R. A. Gottscho, N. Sadeghi, D. J. Trevor, R. W. Boswell, A. J. Perry, T. C. Lee, K. P. Giapis, and J. Margot, Oyo Buturi, Jpn. Soc. Appl. Phys. 61, 711 (1992).

${ }^{15}$ N. Sadeghi, T. Nakano, D. J. Trevor, and R. A. Gottscho, J. Appl. Phys. 70, 2552 (1991); N. Sadeghi, T. Nakano, D. J. Trevor, and R. A. Gottscho, ibid. 71, 3648 (1992); D. J. Trevor, N. Sadeghi, T. Nakano, J. Derouard, R. A. Gottscho, P. D. Foo, and J. M. Cook, Appl. Phys. Lett. 57, 1188 (1990); T. Nakano, N. Sadeghi, and R. A. Gottscho, ibid. 58, 458 (1991).

${ }^{16}$ T. Nakano, N. Sadeghi, D. J. Trevor, R. A. Gottscho, and R. W. Boswell, J. Appl. Phys. 72, 3384 (1992).

${ }^{17}$ E. A. Den Hartog, H. Persing, and R. C. Woods, Appl. Phys. Lett. 57, 661 (1990); R. C. Woods, R. L. McClain, L. J. Mahoney, E. A. Den Hartog, H. Persing, and J. S. Hamers, SPIE 1594, 366 (1991); E. A. Den Hartog, H. Persing, J. S. Hamers, and R. C. Woods, 10th Int. Symp. on Plasma Chem., Bochum, Germany, August 4-9, 1991; E. A. Den Hartog, H. Persing, J. S. Hamers, and R. Claude Woods, 44th Gaseous Electronics Conference, Albuquerque, NM, October 1991, Abstract DA-1.

${ }^{18}$ S. Samukawa, Jpn. J. Appl. Phys. 30, 3154 (1991); S. Samukawa, Jpn. J. Appl. Phys. 30, L1902 (1991).
${ }^{19}$ C. T. Gabriel and J. P. McVittic, Solid State Technol. 35 (June), 81 (1992).

${ }^{20} \mathrm{~K}$. Tsunokuni, K. Nojiri, S. Kuboshima, and K. Hirobe, Ext. Abstr. 19th Conf. Solid State Dev. and Materials, Tokyo, 1987, p. 195.

${ }^{21}$ A. J. Perry and R. W. Boswell, Appl. Phys. Lett. 55, 148 (1989); R. W. Boswell, Ph.D. thesis, Flinders University of South Australia, July 1970; R. W. Boswell, Phys. Lett. 33A, 457 (1970); P. K. Lowenhardt, B. D. Blackwell, R. W. Boswell, G. D. Conway, and S. M. Hamberger, Phys. Rev. Lett. 67, 2792 (1991).

${ }^{22}$ T. Shoji, IPPJ Annual Review, Nagoya University, p. 83 (1988); T. Shoji: IPPJ Annual Review, Nagoya University, p. 67 (1986); A. Komori, T. Shoji, K. Miyamoto, J. Kawai, and K. Kawai, Phys. Fluids B 3, 893 (1991); T. Shoji, IPPJ Annual Review, Nagoya University, p. 63 (1987).

${ }^{23}$ R. Bowers, C. Legendy, and F. E. Rose, Phys. Rev. Lett. 7, 339 (1961).

${ }^{24}$ G. N. Harding and P. C. Thonemann, Proc. Phys. Soc. 85, 301 (1965).

${ }^{25}$ L. C. Woods, J. Fluid Mech. 13, 570 (1962).

${ }^{26} \mathrm{~K}$ losenberg, McNamara, and Thonemann, J. Fluid Mech. 21, 545 (1965).

${ }^{27}$ F. F. Chen, Laser Part. Beams 7, 551 (1989); F. F. Chen, Plasma Phys. Contr. Fusion 33, 339 (1991).

${ }^{28}$ S. Gerstenkorn and P. Luc, Atlas du Spectre d'Absorption de la Molecule d'Iode (CNRS, Paris, 1978); S. Gerstenkorn and P. Luc, Rev. Phys. Appliq. 14, 791 (1979).

${ }^{29}$ A. C. G. Mitchell and M. W. Zemansky, Resonance Radiation and Excited Atoms (Cambridge University Press, Cambridge, England, 1971).

${ }^{30}$ J. Sabbagh and N. Sadeghi, J. Quantum Spectrosc. Rad. Transfer. 17, 297 (1977).

${ }^{31}$ N. Sadeghi, Ph.D. thesis, Universite Scientifique et Medicale de Grenoble, 1974

${ }^{32}$ R. C. Wamsley, J. E. Lawler, J. H. Ingold, L. Bigio, and V. D. Roberts, Appl. Phys. Lett. 57, 2416 (1990).

${ }^{33}$ J. E. Lawler, E. A. Den Hartog, and W. N. G. Hitchon, Phys. Rev. A 43, 4427 (1991).

${ }^{34}$ G. R. Scheller, R. A. Gottscho, D. B. Graves, and T. Intrator, J. Appl. Phys. 64, 598 (1988).

${ }^{35}$ J. P. Booth, O. Joubert, J. Pelletier, and N. Sadeghi, J. Appl. Phys. 69, 618 (1991); P. Baltayan, F. Hartmann, I. Hikmet, and N. Sadeghi, J. Chem. Phys. 97, 5417 (1992).

${ }^{36}$ Oscillator strengths are calculated from the corresponding Einstein A coefficients (see Ref. 29) taken from V. Vujnovic and W. L. Wiese, J. Phys. Chem. Ref. Data 21, 919 (1992).

${ }^{37}$ Although to our knowledge no observations of it have been reported, the ${ }^{4} G$ state arising from the $3 p^{4} 3 d$ electron configuration cortainly exists and should be metastable. The corresponding ${ }^{4} D$ and ${ }^{4} F$ states are approximately 18000 and $7000 \mathrm{~cm}^{-1}$ below their respective doublets so we expect the ${ }^{4} G$ state to lie roughly $2000-3000 \mathrm{~cm}^{-1}$ below the ${ }^{2} G$ state. Except for the ground ionic state, all the states below the ${ }^{2} G_{9 / 2}$ level have even parity and, therefore, can only radiate to the ground state via an electric dipole transition. Since the ${ }^{2} G$ state itself is metastable, it is reasonable to conclude that the unseen ${ }^{4} G$ state is also metastable.

${ }^{38}$ K. Tsujimoto, S. Tachi, S. Arai, H. Kawakami, and S. Okudaira, Dry Process Symposium, Tokyo 1988, p. 42.

${ }^{39}$ S. Samukawa, S. Mori, and M. Sasaki, Jpn. J. Appl. Phys. 29, 792 (1990).

${ }^{40}$ R. A. Dandl and G. E. Guest, J. Vac. Sci. Technol. A 9, 3119 (1991).

${ }^{41}$ Closer examination of the data in Fig. 7 will reveal that the scaling of $n_{m}$ with electron density is actually greater than $n_{e}^{2}$, particularly at the higher pressure. The most likely explanation for such deviation is an increase in $T_{e}$ with if power. Thus, the formation rate coefficients will increase along with the electron density and the overall scaling will exceed the $n_{e}^{2}$ dependence expected for a 2 -step process. It is conceivable that some 3 -step processes may also play a role in forming the metastable ion states.

${ }^{42}$ The neutral metastable density can also be significant. Using a small resonance lamp, we measure absorption by the ${ }^{3} P_{2}$ metastable state at $696.5 \mathrm{~nm}$ and estimate the line-averaged density to be $2.6 \times 10^{10} \mathrm{~cm}^{-3}$. 